\title{
Secondary Instabilities of Surface Waves on Viscous Fluids in the Faraday Instability
}

\author{
Laurent Daudet, Valérie Ego, Sébastien Manneville, and John Bechhoefer \\ Department of Physics, Simon Fraser University, Burnaby, B.C., V5A 1 S6 Canada
}

\begin{abstract}
Secondary instabilities of Faraday waves show three regimes: (1) As seen previously, low-viscosity $(\nu)$ fluids destabilize first into squares. At higher driving accelerations $a$, squares show low-frequency modulations corresponding to the motion of phase defects, while theory predicts a stationary transverse amplitude modulation (TAM). (2) High- $\nu$ fluids destabilize first to stripes. Stripes then show an oscillatory TAM whose frequency is incommensurate with the driving frequency. At higher $a$, the TAM undergoes a phase instability. At still higher $a$, edge dislocations form and fluid droplets are ejected. (3) Intermediate- $\nu$ fluids show a complex coexistence of squares and stripes, as well as stationary and oscillatory TAM instabilities of the stripes.
\end{abstract}

47.20.-k, 47.35.+i 


\section{INTRODUCTION}

Over the past fifteen years, the problem of pattern formation and dynamics in nonequilibrium, spatially extended systems has attracted much attention. Although the most thorough and precise work has been on Rayleigh-Bénard Convection (RBC) and TaylorVortex flow, another hydrodynamic system, the Faraday instability [1], shows great promise. Here, surface waves are excited on a fluid by vertically vibrating its container. The instability is due to parametric resonance: in the reference frame moving with the container, gravity is modulated periodically at a frequency $\omega=2 \pi f$, leading to surface waves vibrating at $\omega_{r}=\omega / 2$. Among the allures of the Faraday instability are its short time scales, large aspect ratios, and small number of material parameters.

Large aspect ratios are important, for distant boundaries can still strongly influence pattern formation [2]. A figure of merit is the aspect ratio $\Gamma \equiv L / \xi$, where $L$ is the system size and $\xi$ the spatial correlation length. In many experiments, $\xi \approx \lambda$, the pattern wavelength. Aspect ratios of order 100 have been achieved recently in RBC [3] and in chemical reactions [4. A number of experiments on the Faraday instability using low-viscosity fluids have been conducted in containers with geometrical aspect ratios $\Gamma^{\prime} \equiv L / \lambda \approx 100$ [5, (6, , [7, 8, 9]. However, as pointed out by Edwards and Fauve [10], the damping length $(\approx \xi)$ of waves in low-viscosity fluids such as water and butanol can be much larger than $\lambda$ (one can have $\Gamma^{\prime} \approx 100$ while $\Gamma \approx 1$ ), implying that patterns in small- $\Gamma$ experiments should be sensitive to details of the boundary conditions well above threshold, even when $\Gamma^{\prime}$ is large. Indeed, they are [1].

In this letter, we report observations of secondary instabilities for varying viscosities. We focus on high-viscosity fluids where $\xi \approx \lambda$, in a box of moderate aspect ratio ( $\Gamma$ ranges up to 12 , depending on $\omega$ ). At high viscosities, the primary instability gives a stripe pattern, in contrast with the squares observed for low viscosities [10, 12]. The sequence of secondary instabilities of stripe patterns is quite different from that observed for squares. At moderate viscosities, the competition between square and stripe states makes the situation more complex still.

In low-viscosity fluids, squares destabilize via low-frequency modulations that Ezersky et al. interpreted as transverse amplitude modulations (TAM) of one component of the two sets of standing waves making up a square pattern [5]. Amplitude equations for standing waves do indeed predict a secondary TAM instability [5, 13, 14. A similar analysis was assumed to hold for square patterns, but Douady [15] showed by stroboscopic methods that the supposed TAM instability was actually a set of kink defects separating two sets of waves $180^{\circ}$ out of phase, an observation that we have confirmed. Here, we show that when the primary instability is a stripe state, the secondary instability is an oscillatory TAM instability. A stationary TAM instability of very small amplitude is, however, seen in moderate-viscosity fluids.

The above concerns control-parameter ramps, where the acceleration of the fluid container is slowly increased from zero. Quite different phenomena, such as converging target patterns and spirals, are observed when the control parameter suddenly jumps from zero to a large value 10,16,17. 


\section{DESCRIPTION OF THE EXPERIMENTAL APPARATUS}

Our apparatus has been described at length in a previous study of acceleration thresholds and onset wavelengths [18]. That study showed that under the proper conditions, we could predict quantitatively thresholds and onset wavelengths using a theory that assumed infinite lateral extent, infinite depth, but finite viscosity 19 .

We used a square sample container of side $L=12.0 \mathrm{~cm}$ and depth $1.0 \mathrm{~cm}$. The fluid temperature could be controlled between 0 and $60{ }^{\circ} \mathrm{C}[20]$, with a stability of $\pm 0.001^{\circ} \mathrm{C}$. Temperature variation due to vertical gradients was $0.5{ }^{\circ} \mathrm{C}$ in the stationary fluid but was less than $0.05{ }^{\circ} \mathrm{C}$ once surface waves appeared. Our control parameters were the driving frequency $\omega$ and the acceleration $a$, which was measured directly by an attached accelerometer. The acceleration could be varied from 0 to $17 \mathrm{~g}$ 's and was regulated to $1 \%$. A spatial homogeneity of $<1 \%$ variation was achieved by careful leveling and dynamic balancing. "Soft" boundary conditions were created by using sloping sidewalls, which greatly reduced wave reflections from the boundaries.

We used three different working fluids: a vacuum-pump oil made of paraffins (Fisher Scientific, Brand 19), propylene glycol, and glycerine. The properties of the pump oil were stable over days and allowed repeatable threshold measurements. Propylene glycol and glycerine could be doped with paint pigment, as described below, and allowed us to scan a wide range of viscosities $\left(0.2-5 \mathrm{~cm}^{2} / \mathrm{sec}\right)$. Polar fluids, however, have much less stable material properties: their surface tension decreases upon contamination by small amounts of impurities, and their viscosity decreases if small amounts of water are absorbed. The sensitivity of the surface tension to impurities implied that different runs for glycerine and propylene glycol samples gave values of the surface tension that differed by as much as $30 \%$. In data quoted below, the material parameters were determined by fits to acceleration thresholds and onset wavelengths. These values were stable to about $5 \%$ over the course of a day. Again, the oil samples did not display such variability. All phenomena observed in the polar fluids were also present in the oil.

We used three methods for visualizing the fluid's surface. Because the waves are very steep (surface inclinations can exceed $90^{\circ}$; see Fig. 1ff), methods based upon specular reflection, such as the shadowgraph technique, cannot give much information about the detailed structure of the waves. (1) We doped the fluid with paint-pigment particles in order to turn the fluid surface into a diffuse light source [21]. We added $2 \%$ (by weight) polymer-stabilized titanium dioxide particles to the glycerine and propylene glycol (the particles could not be suspended in the pump oil). We then illuminated the fluid surface using a stroboscope whose light was directed from the side of the container at an angle that minimized specular reflection into the CCD camera. (See Fig. 1.) (2) We projected a known shape (straight edge) onto the pigment-doped fluid surface. From its apparent form, we could infer the surface profile. (3) We suspended a ring of LEDs above the container [18]. The LEDs were strobed with millisecond pulses and gave the positions of wave peaks and troughs. This method showed that all of the phenomena observed in glycerine and propylene glycol (with or without the paint particles) were also present in the oil. 


\section{EXPERIMENTS}

Figs. 1 1 and 2 summarize the patterns seen in high-viscosity fluids when the acceleration is increased slowly from zero. Below a critical acceleration $a_{c 1}$, the surface remained flat. Just above $a_{c 1}$, for $\varepsilon \equiv\left(a-a_{c 1}\right) / a_{c 1} \lesssim 10^{-2}$, we observed complicated, slowly shifting patterns (Fig. 11a). Because the bifurcation away from a flat interface is (nearly) supercritical [22], the correlation length is large when $\varepsilon \approx 0$. (One expects $\xi \sim \xi_{0}|\varepsilon|^{-1 / 2}$, where $\xi_{0}$ is the correlation length far from the transition.) In Fig. 1 $1 \mathrm{a}, \xi \approx L .(\Gamma \approx 1$.) The effects of spatial gradients in $\varepsilon$ are important in this region, further complicating the observed patterns.

At higher $a$, the surface waves spontaneously order into standing waves of stripes when $\Gamma \gtrsim 3$. (The $\Gamma$-value where stripes order depends on the boundary conditions.) (Fig. 1 b b). In different runs, the stripes were parallel to one or the other of the sides of the square container.

Above a second acceleration threshold, $a_{c 2}$ (the value of $\varepsilon$ depends greatly on $\omega$ ), we observed a supercritical bifurcation to an oscillatory TAM instability of the stripe state. (Fig. 11c). In one side of the cell, left-going traveling TAM waves dominate; in the other side, right-going TAM waves dominate. In the center, the modulations formed a standing wave. The frequency $\omega_{T A M}$ of the standing wave was incommensurate with the driving frequency $f$. (See Fig. 3.) The TAM frequency depends approximately linearly on $f$, but systematic deviations are clearly seen. The solid line in Fig. 3 shows a quadratic fit, which needs theoretical justification. The wavelength of the TAM was $(3.30 \pm 0.35) \lambda$ over the range of frequencies studied; the ratio $\omega / \omega_{T A M}$ varied from 5.15 to 5.39 (i.e., $\omega_{r} / \omega_{T A M} \approx 2.58$ to 2.70).

At a third acceleration threshold, $a_{c 3}$, we observed the spontaneous formation of defects. The line in Fig. 2 represents the acceleration where isolated single defects - edge dislocations - first appeared spontaneously. (Fig. 11e.) They moved to the cell edge by a combination of climb and glide. Above $a_{c 3}$, the number of defects increased rapidly, resulting in very complicated dynamics. (Fig. 1ff.) We have not analyzed this regime, but it might be useful to connect it with studies of defect-mediated turbulence [2]. In the defect regime, droplets of fluid were spontaneously ejected upwards. Droplet emission was correlated with pattern defects, and their frequency rapidly increased with $\varepsilon$.

Between $a_{c 2}$ and $a_{c 3}$, there is clear evidence for another threshold where the TAM modulations dephase (Fig. 11d.) However, the dynamics of this regime are complicated, and our cell was too small to study this tertiary instability quantitatively.

We also investigated the effect of changing viscosity by changing the temperature of propylene glycol and glycerine samples. At $50 \mathrm{~Hz}$ and $\gamma / \rho=35 \mathrm{~cm}^{3} / \mathrm{sec}^{2}$, for example, we found that in propylene glycol, squares are stable for $\nu \lesssim 0.5 \mathrm{~cm}^{2} / \mathrm{sec}$ while stripes are stable for $\nu \gtrsim 0.9 \mathrm{~cm}^{2} / \mathrm{sec}$. The crossover region between squares and stripes is complex and deserves careful study in its own right. For example, in the same fluid sample, at $\nu$ $=0.63 \mathrm{~cm}^{2} / \mathrm{sec}$, we destabilize first to a square state and then, at $\varepsilon=0.28$, to a timedependent, probably chaotic state. At $\varepsilon=0.34$, this state gives way to a stripe state with stationary TAM modulations. At $\varepsilon=0.44$, the oscillatory TAM sets in. The wavelength of the stationary TAM is 1.4 times the stripe wavelength $\lambda$, while that of the oscillatory TAM is, as mentioned above, 3.3 $\lambda$. Also, by creating a stripe state at high viscosity and then slowly decreasing the temperature of the fluid, it is possible to preserve the state 
even at temperatures where ramping from below threshold leads unambiguously to squares. (The reverse is true, as well.) The crossover region is also distinguished by low-frequency dynamics for $a \approx a_{c 1}$, driven presumably by competition between the two primary states. In this regime, we confirmed that stripe states are born with a stationary TAM and then further destabilize via oscillatory TAMs at higher $\varepsilon$, while square states show $180^{\circ}$ phase defects. A promising feature of the intermediate-viscosity regime is the possibility, in a larger-aspect cell, of finding a spatiotemporally chaotic state at the onset of the primary instability [17].

\section{DISCUSSION}

Secondary instabilities in viscous fluids that have stripe patterns differ markedly from those observed in the low-viscosity, square-pattern case. The TAM instability predicted for the latter is in fact only observed for the former. While theory predicts a stationary TAM, the instability is oscillatory with a frequency that is generically incommensurate with the driving frequency. In the crossover regime, both stationary and oscillatory TAMs are seen. It would be interesting to explore the case where $\omega_{T A M}$ is commensurate with $\omega_{r}$. The closest integer ratio, $\omega_{r}=3 \omega_{T A M}$, requires higher combinations of $\omega$ and $a$ than were possible with our shaker table.

An obvious theoretical challenge is to see whether the four coupled amplitude equations describing standing waves can account for the oscillatory TAM instability. The coefficients in the amplitude equations were derived assuming small damping and small surface slopes [13]. Neither of these conditions is met for high-viscosity fluids [23], although the approximations are more reasonable for fluids of moderate viscosity. This suggests difficulties for perturbation expansions based on a flat interface and small damping, but numerical studies remain feasible, as do studies based on models analogous to the Swift-Hohenberg equation [2.14. In the intermediate-damping regime, a small number of amplitude equations may adequately describe what appear to be spatiotemporally chaotic states. Such a situation has recently been discovered in a rotationally anisotropic system [24], but no isotropic system has heretofore been found.

All of these phenomena - tertiary phase instabilities, locking between primary and secondary instabilities, square-stripe competition, the crossover regime — will be much easier to explore if one can increase the aspect ratio. Since much larger shaker tables (albeit expensive) are available commercially, there is no technical limitation to increasing the aspect ratio at least tenfold.

\section{ACKNOWLEDGMENTS}

We thank A. Rawicz for the loan of the vibration exciter and W. González-Viñas for help with many aspects of the experiment. This work was supported by NSERC (Canada). J. B. is an Alfred P. Sloan Foundation Fellow. L. D., V. E. and S. M. acknowledge support from the ENS (Paris). 


\section{REFERENCES}

[1] Faraday M., Phil. Trans. R. Soc. Lond. 52319 (1831).

[2] Cross M. C. and Hohenberg P. C., Rev. Mod. Phys. 65851 (1993).

[3] Morris S. W., Bodenshatz E., Cannell D. S., and Ahlers G., Phys. Rev. Lett. 712026 (1993); Assenheimer M. and Steinberg V., Phys. Rev. Lett. 703888 (1993).

[4] Ouyang Q. and Swinney H. L., Nature 352610 (1991).

[5] Ezersky A. B., Rabinovich M. I., Reutov V. P., and Starobinets I. M., Sov. Phys. JETP 641228 (1986).

[6] Ezersky A. B., Ermoshin D. A., and Kiyashko S. V. Phys. Rev. E 514411 (1995).

[7] Gluckman B. J., Arnold C. B., and Gollub J. P., Phys. Rev. E 511128 (1995).

[8] Bosch E., Lambermont H., and van de Water W., Phys. Rev. E 49 R3580 (1994).

[9] Christiansen B., Alstrøm P., and Levinsen M. T., Phys. Rev. Lett. 682157 (1992).

[10] Edwards W. S. and Fauve S., Phys. Rev. E 47 R788 (1993); J. Fluid Mech. 278123 (1994).

[11] A more familiar example of an instability with $\xi \gg \lambda$ well above threshold is the laser: even though the cavity may be millions of wavelengths long, it determines the output modes. For low-viscosity surface waves, the sensitivity to boundary conditions for $\Gamma \approx 1$ but $\Gamma^{\prime} \gg 1$ is clearly seen in time averages of images taken in nominally chaotic regimes [7].

[12] Fauve S., Kumar K., Laroche C., Beysens D. and Garrabos Y., Phys. Rev. Lett. 683160 (1992).

[13] Milner S. T., J. Fluid Mech. 22581 (1991).

[14] Zhang W. and Viñals J., Phys. Rev. Lett. 74690 (1995).

[15] Douady S., J. Fluid Mech. 221383 (1990).

[16] L. Korzinov, private communication.

[17] Daudet L. and Bechhoefer J., unpublished.

[18] Bechhoefer J., Ego V., Manneville S., and Johnson B., J. Fluid Mech. 288325 (1995). Material parameters for the pump oil are given here.

[19] Kumar K. and Tuckerman L. S., J. Fluid Mech. 27949 (1994); Beyer J. and Friedrich R., Phys. Rev. E 51, 1162 (1995). Our calculations also took into account finite depth, but its effects were negligible.

[20] $\nu$ is a strong function of $T$, while $\gamma / \rho$ depends only weakly on $T$.

[21] Faraday noted that opaque fluids such as milk improve the visualization [1].

[22] The bifurcation to squares is supercritical [15]; that to stripes may be slightly subcritical, but more precise measurements are needed to confirm this.

[23] For $\nu=1.0 \mathrm{~cm}^{2} / \mathrm{sec}, k=5.71 \mathrm{~cm}^{-1}, f=42 \mathrm{~Hz}$, we have $\sigma \equiv 4 \nu k^{2} / \omega \approx 0.5$. Perturbation expansions assume $\sigma \ll 1$. Physically, $\sigma$ is the ratio of the period of the waves to their decay time; it is also the inverse of the waves' quality factor. Using our second visualization method, we find that at $a_{c 2}$, the stripe profile $\psi(x) \approx \alpha[\cos (k x)-0.23 \cos (2 k x)]$, implying a maximum surface slope of $80^{\circ}$.

[24] Dennin M., Cannell D. S., and Ahlers G., Mol. Cryst. Liq. Cryst. 261377 (1995). 


\section{FIGURES}

FIG. 1. Patterns observed as the acceleration is ramped slowly from zero. The pictures are strobed to show the maximum stripe amplitude. (a) Primary instability at onset. The effect of gradients here is significant. (b) Stripe instability. (c) Stripes plus oscillatory TAM instability. (d) Phase instability of TAMs. (e) Single defect present in stripe state. (f) Turbulent defects and breaking of surface. Glycerine, $44{ }^{\circ} \mathrm{C}$; depth $h=1.0 \mathrm{~cm} ; \gamma / \rho=25 \mathrm{~cm}^{3} / \mathrm{sec}^{2} ; \nu=1.4 \mathrm{~cm}^{2} / \mathrm{sec} ; f$ $=40 \mathrm{~Hz}, a_{c}=6.1 \mathrm{~cm}^{2} / \mathrm{sec} ; \lambda=1.1 \mathrm{~cm} . \Gamma=L / \xi$ where the correlation length $\xi$ is computed using the range of linearly unstable wavelengths (see [18]). The estimate of $\xi$ assumes $\varepsilon \ll 1$, which may not be valid for the higher values of $\varepsilon$ here.

FIG. 2. Morphology diagram of patterns observed as the acceleration is ramped slowly from zero. Regions corresponding to images shown in Fig. 1 are shown. Oil, $22{ }^{\circ} \mathrm{C}$. Similar data was obtained using glycerine and propylene glycol.

FIG. 3. Frequency of the secondary instability. Glycerine, $45{ }^{\circ} \mathrm{C}, \nu=1.2 \mathrm{~cm}^{2} / \mathrm{sec}, \gamma / \rho=30$ $\mathrm{cm}^{3} / \mathrm{sec}^{2}$. The data are also well fit by $f_{T A M}=a+b f$. 

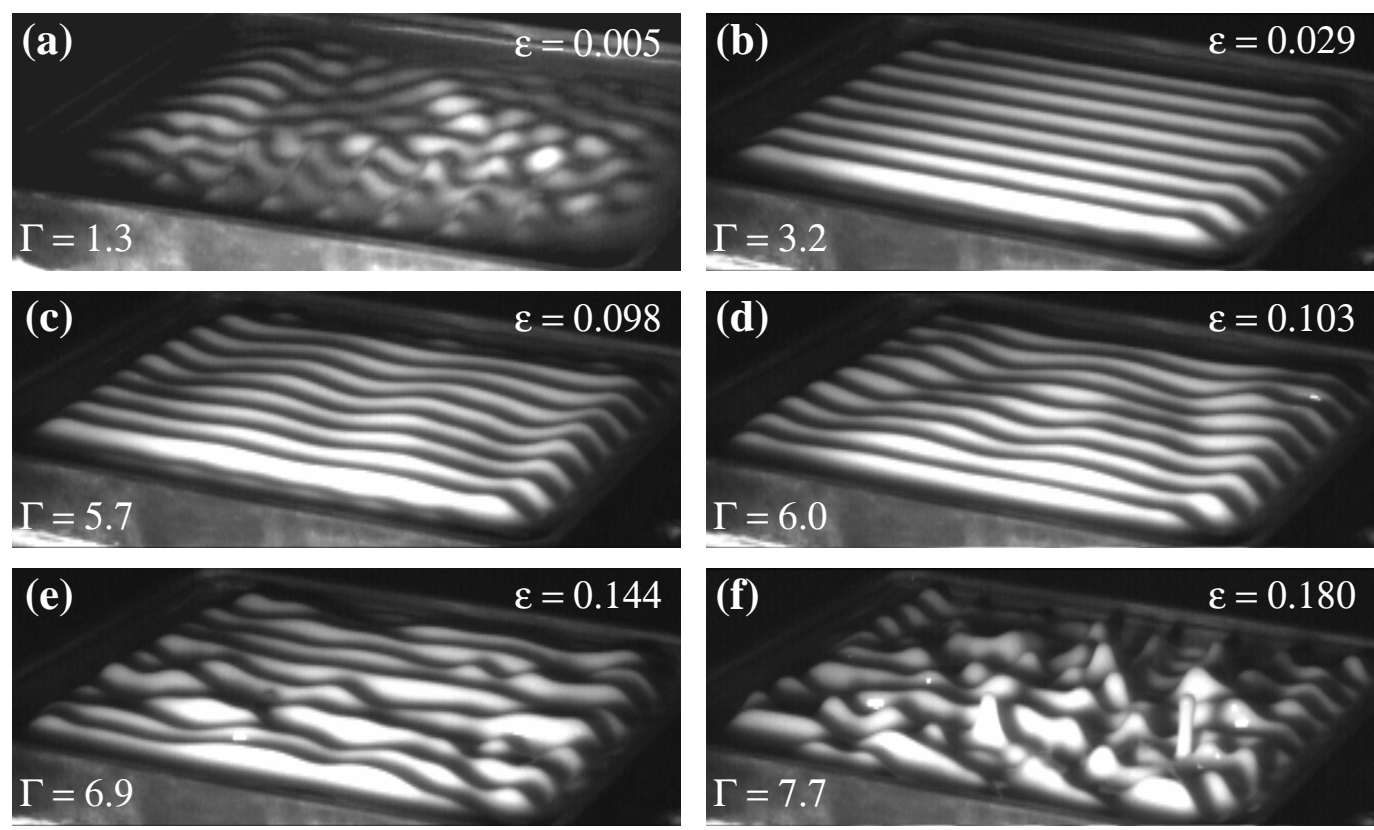

Daudet, Fig. 1 


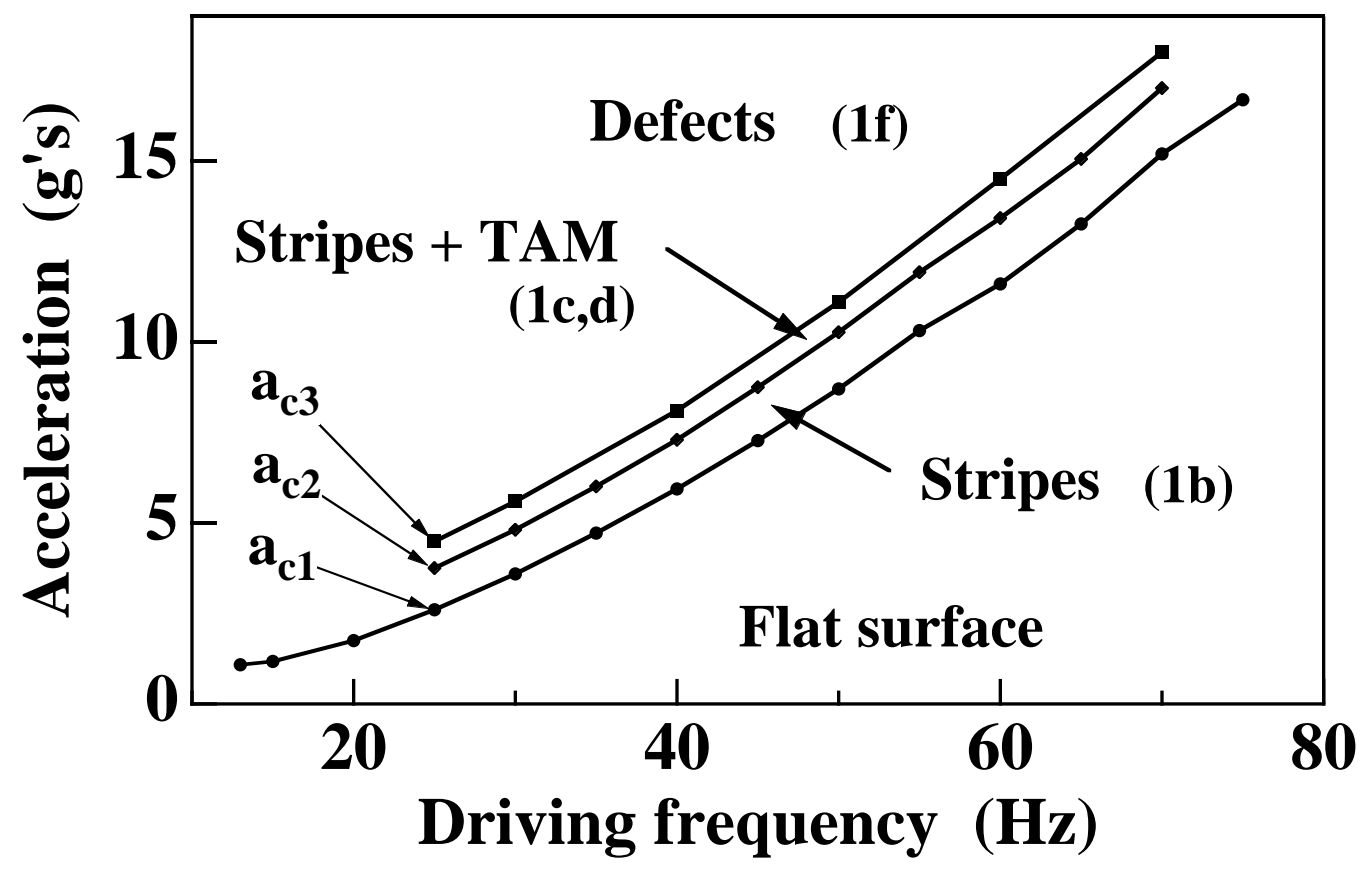

Daudet, Fig. 2 


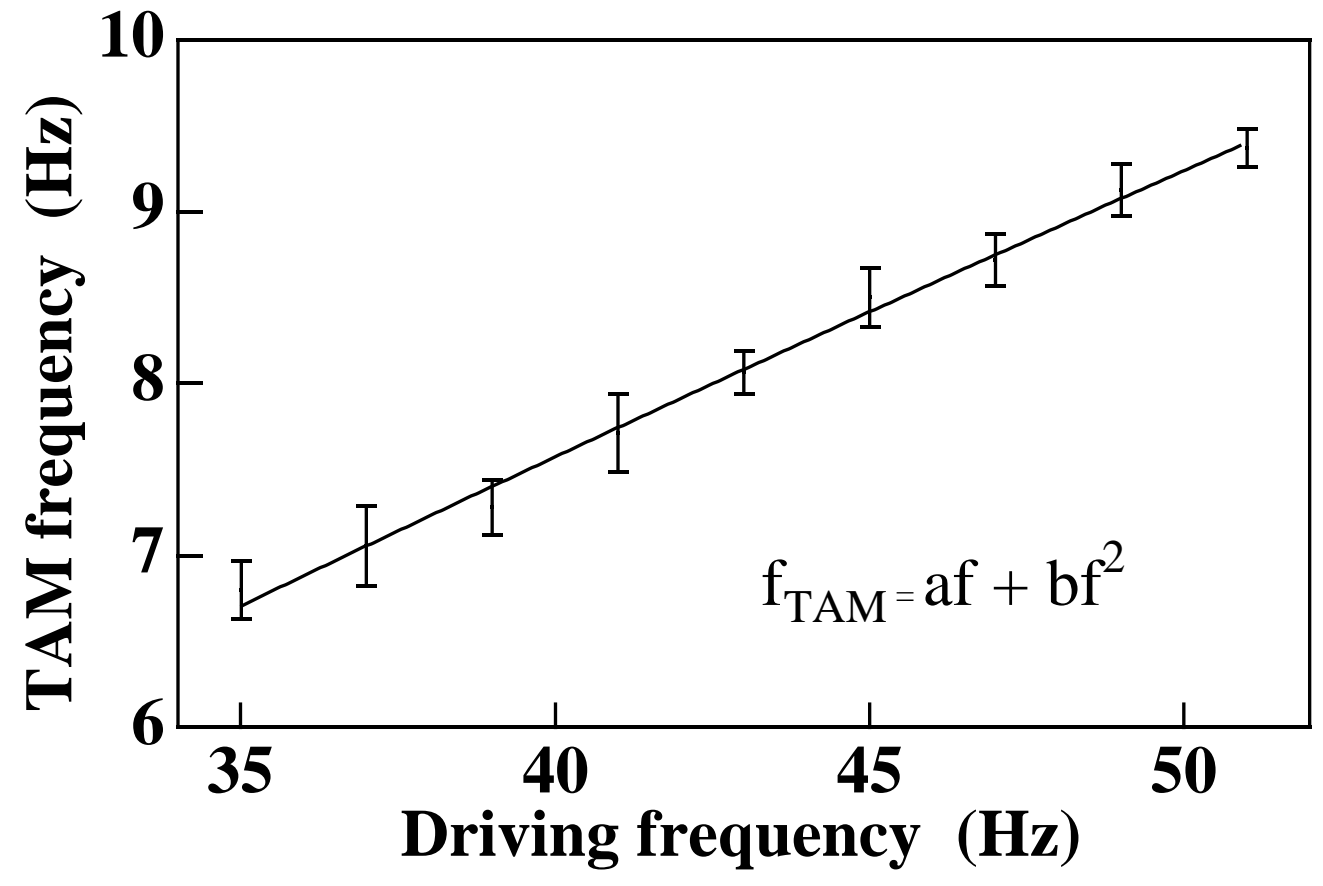

Daudet, Fig. 3 